\title{
Téoros
}

Revue de recherche en tourisme

\section{Tourisme et perspectives de développement au Maroc}

\section{Rachida Saïgh Bousta}

Volume 24, numéro 1, printemps 2005

Maroc, réalités et défis

URI : https://id.erudit.org/iderudit/1071102ar

DOI : https://doi.org/10.7202/1071102ar

Aller au sommaire du numéro

Éditeur(s)

Université du Québec à Montréal

ISSN

0712-8657 (imprimé)

1923-2705 (numérique)

Découvrir la revue

Citer ce document

Bousta, R. S. (2005). Tourisme et perspectives de développement au Maroc.

Téoros, 24(1), 3-5. https://doi.org/10.7202/1071102ar d'utilisation que vous pouvez consulter en ligne.

https://apropos.erudit.org/fr/usagers/politique-dutilisation/ 


\section{Tourisme et perspectives de développement au Maroc}

\section{Rachida Saïgh Bousta}

Plus que jamais, le tourisme est érigé comme une priorité stratégique et une «locomotive de développement » des ressources économiques au Maroc. L'objectif d'atteindre 10 millions de touristes à I'horizon 2010 mise aussi bien sur la nécessité de créer des emplois que sur la possibilité de générer des recettes et des devises. Cet objectif intervient dans une situation où la mondialisation est à la fois un contexte incontournable et un enjeu de taille. Désormais, on se doit de mobiliser les synergies en termes de réflexion et de réalisations innovantes dans un cadre de professionnalisation. II s'agit d'œuvrer pour une véritable démarche créative qui souscrit aux nouvelles données de la concurrence ainsi qu'aux exigences des tendances.

En prenant en compte l'évolution des approches et des stratégies économiques - avec toutes les mutations qui ne cessent de s'opérer sur le paysage international -, vu l'impact des interactions conjoncturelles si péremptoires, le tourisme est indéniablement une valeur, mais une valeur d'une fragilité et d'une vulnérabilité parfois démesurées. Plus que jamais, le secteur appelle à la vigilance eu égard au contexte qui l'engendre et l'accompagne. L'activité touristique exige de plus en plus adaptation et adaptabilité aux interactions avec les secteurs mitoyens qui lui servent de plate-forme culturelle et de canevas socioéconomique; elle fustige la reconsidération des perspectives et des visées liées à l'environnement et à la durabilité.

II est certain que le présent dossier ne peut apporter des réponses à toutes les problématiques que posent les perspectives de développement du secteur touristique au Maroc avec toutes les composantes, les po-

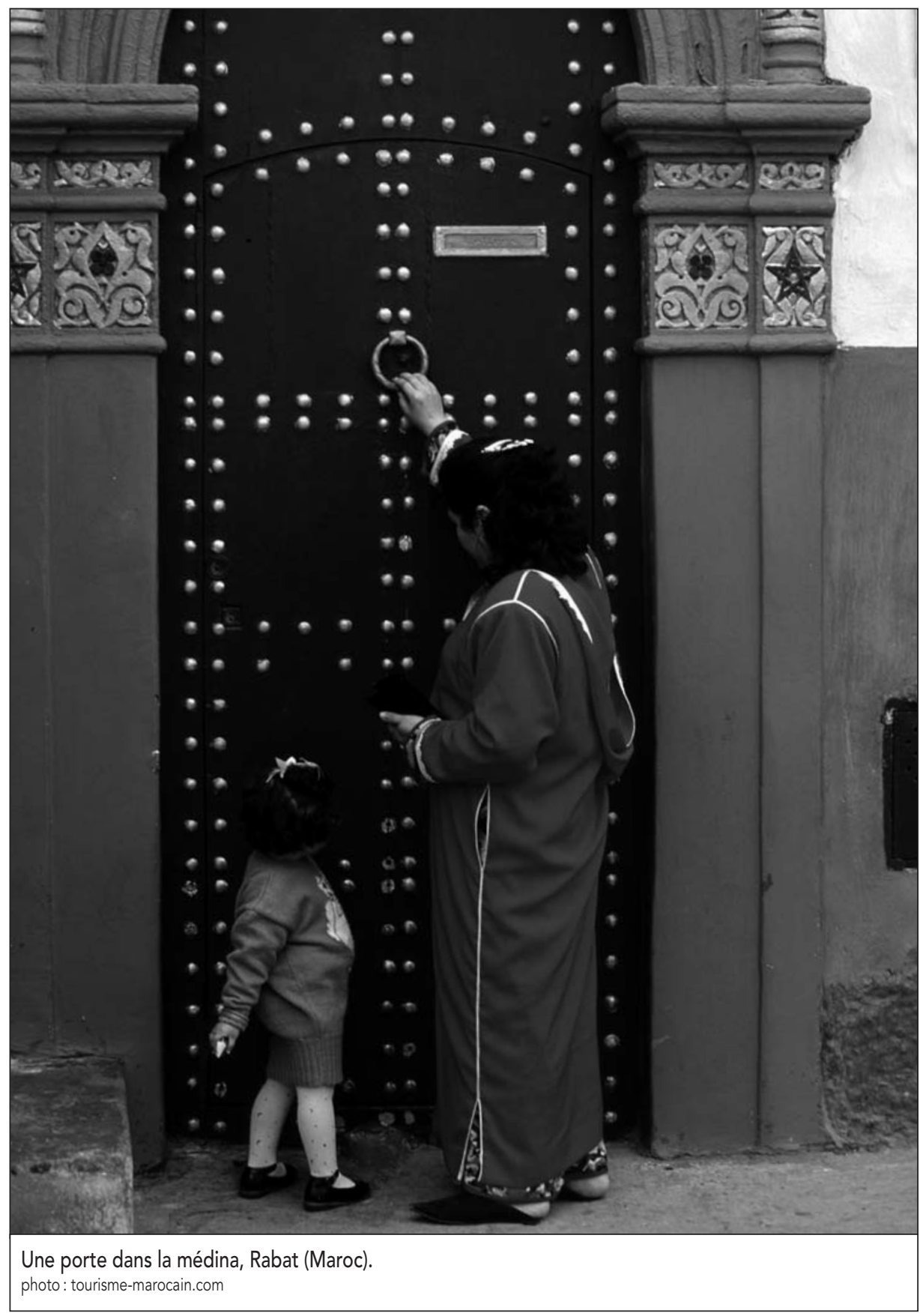


tentiels et l'offre et la demande... II propose cependant quelques lectures et réflexions prospectives sur les réalités et les défis qui accompagnent les stratégies du tourisme au Maroc en mettant l'accent sur les impératifs de la durabilité comme levier de toute conception qui mise sur l'avenir.

Pour aborder une telle problématique, dans une première approche analytique de la situation plus ou moins rétrospective, Mimoun Hilali propose une analyse des stratégies à partir des «Aspects sociogéographiques du développement du tourisme balnéaire au Maroc ", axe qui représente à nouveau l'un des enjeux les plus importants de la «Vision 2010 ». En s'ouvrant à cette activité en plein boom touristique des années 1960, le Maroc a préféré le libéralisme. II a privilégié alors l'option balnéaire tout en finançant des aménagements volontaristes, paradoxalement, à coups de fonds publics. Quarante ans plus tard, constatant que l'intervention massive de l'État n'apporte pas nécessairement les résultats escomptés (Tanger, Tétouan et Al Hoceima), il opte pour une stratégie de «contrat-programme» entre l'État et les acteurs socioéconomiques de première envergure. Cette option part d'un constat rétrospectif : les stations peu (ou pas) concernées par l'action étatique (Agadir, Marrakech, Ouarzazate...) ont remarquablement honoré leurs objectifs. Aussi, la Vision 2010 remet-elle à l'honneur le tourisme balnéaire, l'État ayant un rôle de planificateur. Le Maroc revient ainsi avec force à l'idée du tourisme moteur de développement.

L'article de Saïd Boujrouf s'inscrit dans le prolongement de cette réflexion et procède par une lecture analytique de son déploiement. II esquisse un tableau des différentes étapes qui ont jalonné la politique touristique en termes d'aménagement du territoire. Ainsi propose-t-il à la fois une réflexion critique sur ce cheminement et des perspectives et des reconsidérations sur l'interaction entre "Tourisme et aménagement du territoire ». Cependant, il ne manque pas de s'interroger sur les «agencements » possibles pour mieux œuvrer dans l'objectif d'un développement harmonieux. Du point de vue du géographe, l'aménagement n'est plus seulement un domaine de réflexion et d'action qui peut réunir un certain nombre d'acteurs; il est surtout un levier de concertation et de partenariat dont le but demeure la réalisation d'une œuvre au service de la communauté. Aussi souligne-t-il les stratégies de

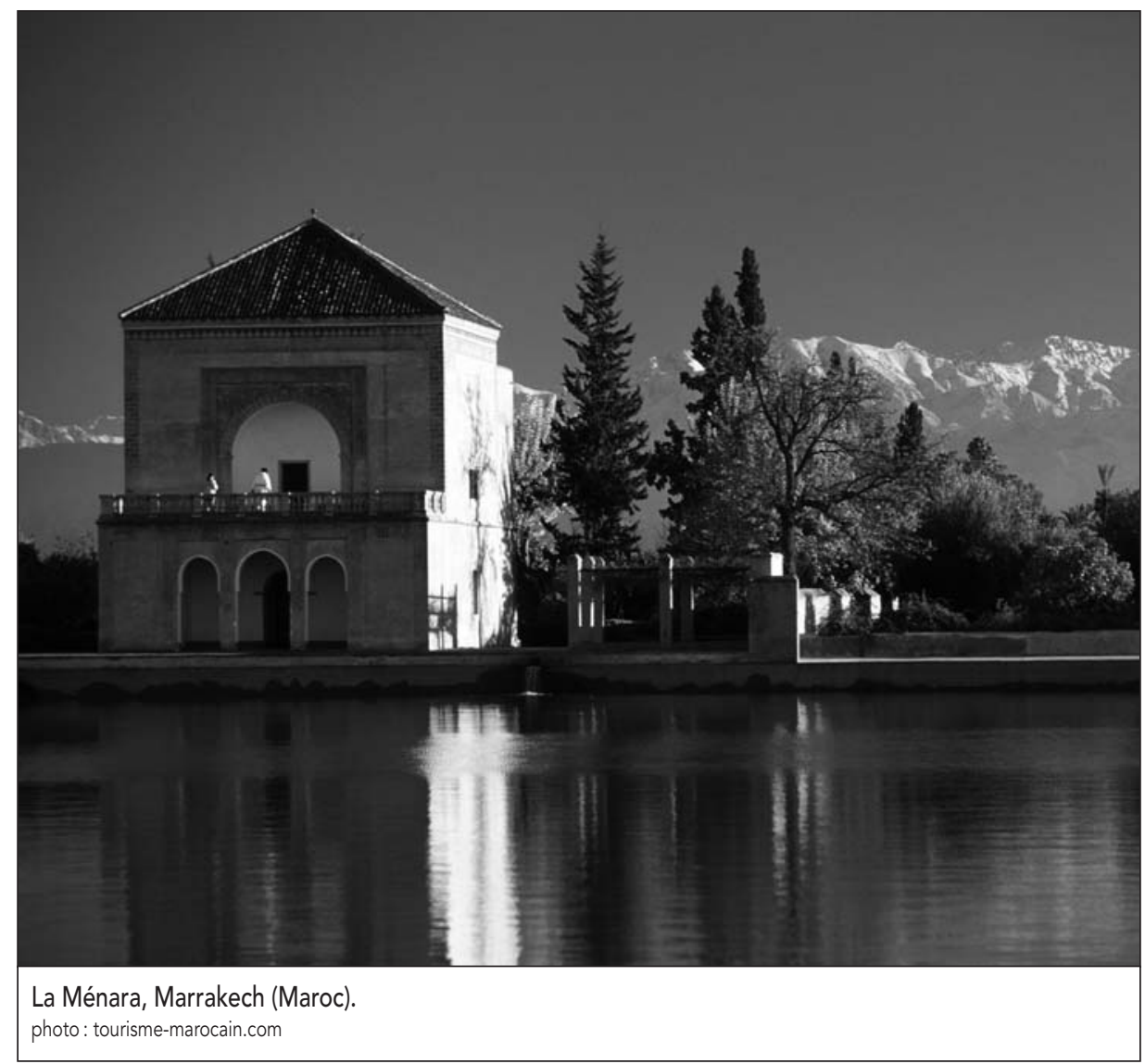

l'approche actuelle qui met en exergue l'intégration de l'entreprise privée et la "société civile ». II s'agit d'une nouvelle politique volontaire de développement et d'aménagement, politique basée sur une approche partenariale et contractuelle qui réunit l'État et d'autres acteurs socioéconomiques.

Cependant, un développement harmonieux et structuré se doit d'intégrer la formation qui l'accompagne et permet de créer une dynamique de qualité aussi indispensable que tous les aménagements et préalables à la mise en œuvre d'une politique de développement réfléchi et concerté. L'article de Fatima Zohra Guertaoui étudie et propose une " contribution de la formation au développement de l'industrie touristique au Maroc ». En effet, la formation professionnelle dans le secteur du tourisme suscite de plus en plus d'intérêt au sein du gouvernement, de la profession et des institutions d'enseignement. Les nouvelles technologies entraînent d'autres formes d'organisation du travail et imposent une amélioration soutenue des aptitudes professionnelles. Cependant, les pratiques de gestion des ressources humaines suscitent encore quelques interrogations dans les métiers du tourisme.
Aussi, les entreprises du secteur sont-elles acculées à intégrer une stratégie de formation et de formation continue. Pour atteindre les objectifs escomptés, il importe de corroborer cette exigence avec le potentiel de sensibilisation et d'organisation des entreprises. L'État pourrait-il s'attribuer le rôle d'incitateur, voire de gardien de la réalisation de la politique de formation? Si la notion de compétence constitue désormais un enjeu capital, une offre de formation optimale et adaptée requiert d'être à l'écoute de la demande des entreprises et, de ce fait, nécessite une intervention sur plusieurs fronts : révision continuelle des programmes, assistance des pouvoirs publics, innovation dans les méthodes d'enseignement, développement de la formation continue et recyclage des formateurs, développement d'associations professionnelles...

L'article de Brahim Moudoud et Abdelkrim Ezaïdi entame une seconde phase dans les études proposées, puisqu'il aborde la problématique selon une approche prospective tournée vers le devenir du tourisme au Maroc. Aussi propose-t-il une ouverture sur «le tourisme national» en envisageant les « opportunités et les limites de développe- 
ment » qui doivent être prises en considération. En effet, le tourisme national présente une aubaine pour le secteur. II offre une option participative au développement de l'économie régionale et locale susceptible d'assurer la survie des entreprises et des hommes en périodes de crise et de basse saison. Les retombées locales pourraient sensiblement contribuer à l'amortissement des investissements, mais force est de constater que ce créneau se développe timidement. Cependant, le Maroc vient d'opter, récemment, pour la promotion de son tourisme intérieur par des campagnes structurées, lesquelles ne font pas l'unanimité d'un grand nombre de structures d'hébergement et de commercialisation. La réussite du tourisme interne supposerait-t-elle l'élaboration d'une stratégie commerciale et d'un plan d'action opérationnel régulier?

L'article de François Vellas et Zouhair Mehadji aborde les "Stratégies touristiques du secteur privé au Maroc ». II part d'un constat fort important. En effet, le Maroc se distingue par le fait qu'il se situe dans une région du monde qui connait le plus fort niveau de développement touristique, avec notamment l'ensemble des pays de la Méditerranée. Or, les produits touristiques offerts par le Maroc sont largement complémentaires de ceux des pays européens. Cette donnée peut favoriser de nouvelles sociétés conjointes entre les investisseurs marocains et internationaux, notamment dans le cadre du Plan Azur. Pour cela, une coopération avec la Royal Air Maroc, en tant qu'investisseur touristique, peut permettre de stimuler et de diversifier la progression de la demande touristique internationale vers le Maroc. Les auteurs ajoutent que, pour apporter une contribution importante au développement touristique, il est nécessaire non seulement de favoriser les programmes traditionnels d'investissements privés dans le tourisme balnéaire, mais aussi de soutenir des micro-projets qui peuvent comporter des réalisations susceptibles de créer un nombre significatif d'emplois. Par conséquent, il est essentiel de réaliser désormais des choix de développement touristique qui, sans aller vers le tourisme de masse, doivent pouvoir combiner la poursuite du développement du tourisme culturel, mais aussi le développement du tourisme d'affaires et la réalisation de grands aménagements du tourisme balnéaire moyen et haut de gamme, pour lesquels les stratégies du secteur privé permettront de relayer les impulsions déterminées par la politique nationale, destinée à faire du tourisme un moteur essentiel de l'économie marocaine.

Dans une approche prospective, Fatima Arib se penche sur une question de grande actualité et d'avenir, à savoir la vision du « tourisme : atout durable du développement au Maroc ». Elle part du principe que l'apport économique de l'activité touristique au Maroc est certes important. Elle souligne le fait qu'il soit de plus en plus considéré comme un secteur stratégique pour accompagner le développement économique. Mais qu'en est-il du coût pour l'environnement et le social ? Le tourisme peut-il être appréhendé comme un facteur de développement durable? Que faut-il pour qu'il joue le rôle essentiel tant attendu? Comment conjuguer la stratégie marocaine de développer et d'accroître quantitativement l'activité touristique avec celle de vouloir faire du tourisme une activité aux retombées durables, sans perte de son potentiel touristique diversifié ? L'objectif d'une telle analyse critique est tourné vers l'exploration des aspects susceptibles de faire du tourisme une activité motrice du développement durable au Maroc.

Après les études à objectifs descriptifs, analytiques et rétrospectifs, à la suite des études prospectives, l'article d'Ahmed Bellaoui nous fait toucher de très près une réalité qui peut être considérée à partir de son exemplarité concrète. Aussi a-t-il choisi de nous présenter un cas précis pour mettre en évidence à la fois une région en phase d'ouverture au tourisme et un sujet de méditation sur son avenir et ses perspectives de durabilité. Son article porte un titre emblématique: " La vallée du Zat, un pays d'accueil touristique émergeant dans l'arrière-pays montagneux de Marrakech ». La question est abordée à partir des "stratégies et des imaginaires du tourisme ". Ahmed Bellaoui se livre à un véritable examen d'un tourisme balbutiant. Bien qu'encore méconnue des touristes, car faiblement médiatisée, la vallée du Zat possède d'importants atouts touristiques au nombre desquels d'authentiques paysages de moyenne et haute montagne. Ainsi, la promotion toute récente de la vallée par des associations locales commence à donner ses fruits, surtout dans les domaines de l'écotourisme, de la randonnée et de la découverte des modes de vie des nombreuses communautés villageoises de la vallée. Quel avenir une telle ouverture augure-t-elle pour le pays et ses habitants?
Avec le souci d'ouvrir d'autres brèches de réflexion sur des exemples palpitants d'actualité et surtout engrossés d'interrogations quant au devenir que peut leur réserver une ouverture plus ou moins brutale sur le tourisme, Ouidad Tebbaa et moi-même avons voulu consacrer un moment de réflexion et de méditation de Téoros à une partie non négligeable du patrimoine de Marrakech. D'où un titre complexe et quelque peu provocateur: "Stratégies et imaginaires du tourisme: cas des ryads maisons d'hôtes et mutations de la médina de Marrakech ». Certes, la culture est de plus en plus exploitée comme une sorte d'étendard pour donner du sens, redoubler les motivations et créer une valeur ajoutée au produit touristique. Les "ryads", vielles demeures de la médina, en sont un exemple emblématique. Mais quel est le prix à payer quand les jeunes marrakchis hypothèquent la mémoire intramuros et succombent aux charmes de la modernité qui s'introduit dans les espaces les moins propices au tourisme? Quelle stratégie utiliser pour faire en sorte que ce terreau puisse continuer à être un espace pérenne permettant à l'autochtone et à l'étranger de se ressourcer dans une culture et une mémoire anciennes? Ces réflexions et ces interrogations ont en amont une enquête effectuée auprès des autochtones qui vivent dans l'entourage immédiat des ryads transformés en maisons d'hôtes.

Enfin, pour clore ce dossier sur le Maroc, Jean Stafford dresse un bilan statistique sur l'état du tourisme au pays depuis les quinze dernières années. L'auteur analyse les fluctuations des arrivées des touristes internationaux et montre que les deux guerres du Golfe ont eu des répercussions significatives sur celles-ci.

Rachida Saïgh Bousta est enseignante-chercheure en culture et patrimoine, directrice de l'École doctorale internationale de tourisme (EDIT), vice-présidente de l'Université Cadi Ayyad et chargée de recherche et de coopération. Elle est rédactrice invitée pour ce dossier. 